\title{
La Gramática Funcional de Dik
}

\section{Dik's Functional Grammar}

Ligia Ochoa Sierra ${ }^{1}$

\section{Resumen}

Este artículo da cuenta del modelo lingüístico denominado gramática funcional de Dik. Este modelo ha sido empleado en los últimos sesenta años para hacer descripciones de varias lenguas. Se pretende presentar una visión sistemática y coherente de este modelo. Para ello, se presentan inicialmente las características generales de lo que se denomina gramática funcional, luego se describen la estructura del modelo y los principios metodológicos; por último, se hace una breve alusión al modelo funcional denominado gramática del rol y la referencia. La revisión se basa principalmente en los trabajos pioneros de Dik.

\section{Palabras clave}

Funcionalismo, gramática de dependencias, gramática funcional, estructura clausal, predicados.

Abstract

This article presents the linguistic model called Dik's «Functional Grammar». This model has been used in the past sixty years to describe various languages. We try to present a systematic and coherent model. In this way, the general characteristics of what is called Functional Grammar are initially presented. Then we are going to describe the structure of the model and the methodological principles, and finally, a brief reference to the functional model called Grammar Role and Reference. The review is mainly based on the pioneering Dik's work.

\section{Key words}

Functionalism, dependency grammar, functional grammar, clausal structure, predicates.

Artículo recibido el 27 de enero de 2011 y aprobado el 19 de agosto de 2011

1 Profesora Departamento de Lingüística de la Universidad Nacional de Colombia. Correo electrónico: lochoas@bt.unal.edu.co 


\section{La gramática funcional}

La gramática funcional de Dik hace parte de lo que se conoce en la bibliografía lingüística con el nombre de gramática funcional. Este rótulo reúne una serie de trabajos [la gramática de valencias, de Tesniere (1959), el estructuralismo funcional de Martinet (1962), la gramática sistémico funcional de Halliday (1967), la gramática funcional de Dik (1978) y la gramática del papel y la referencia de Van Valin (1993)] que tienen como común denominador el hecho de considerar que la función principal del lenguaje es la comunicación entre los seres humanos. La lengua es definida como un instrumento de interacción social, usada con el objetivo primordial de establecer relaciones de comunicación entre hablantes y destinatarios (Dik, 1997, p. 5). Por lo tanto, requisitos pragmáticos de la interacción verbal guían la descripción de las lenguas y lo hacen, como lo señala García (2003, p. 62), sin renunciar a un alto grado de formalización.

También es común a todos los modelos que se agrupan bajo esta denominación, la relación de dependencia que se establece entre el núcleo (predicado) y sus constituyentes obligatorios, la estructura jerárquica de la cláusula y la atención que prestan al componente léxico de la lengua. Asimismo, todas las teorías funcionales parten de los datos lingüísticos perceptibles y emplean métodos inductivos para la construcción de la teoría.

Pueden considerarse como pioneros de la gramática funcional los trabajos de Tesniére, Martinet, Halliday y Fillmore. Así, Tesniere (1959) introduce la noción de valencia y con ello las relaciones de dependencia que se dan al interior de las lenguas; Martinet (1962) introdujo la noción de enunciado mínimo (núcleo + constituyentes obligatorios) y la concepción de la lengua como un proceso continuo de adaptación a las necesidades comunicativas. Halliday (1985) destaca el carácter social del lenguaje, la gramática paradigmática o lexical y las tres funciones que determinan el modelo lingüístico: función ideativa (el lenguaje como representación de la realidad), interpersonal (el lenguaje como mediador de las relaciones personales) y textual (el lenguaje como medio para referirse a él mismo), siguiendo de esta manera la línea de los lingüistas de Praga. Por su parte, fue Fillmore quien, a través de la teoría de los casos, puso de relieve las funciones semánticas, esenciales para una gramática funcional, ya que el componente semántico determina en gran medida el nivel sintáctico e incluso pragmático. Para los lingüistas funcionalistas, la noción de función atraviesa los tres componentes de la lengua y determina en gran medida la estructura de los distintos modelos lingüísticos. Así, en las propuestas funcionales se distinguen funciones sintácticas, semánticas y pragmáticas. Para Dik (1997), las funciones sintácticas expresan la perspectiva desde la que se presenta el estado de cosas en la expresión lingüística (sujeto y objeto); las funciones semánticas especifican los papeles que representan los referentes de los términos implicados en el estado de cosas designado por la predicción en que aparecen (agente, paciente, instrumento, etc.) y las funciones pragmáticas especifican el estatus informativo de los constituyentes dentro de la más amplia localización comunicativa en que aparecen: (tópico, foco).

\section{La Gramática funcional de Dik}

De acuerdo con el principio funcional de que la lengua es un instrumento de comunicación, el componente gramatical de la gramática funcional de Dik (GF) se integra en una teoría de la interacción verbal, y en este sentido se puede afirmar que la función representativa del lenguaje se integra en la función interpersonal o social. Como consecuencia de esta concepción, Dik (1978)

considera que la lingüística debe ocuparse de al menos dos sistemas de reglas, ambos de naturaleza social: las reglas que rigen la interacción verbal como una forma de actividad cooperativa (reglas pragmáticas) y las reglas que rigen las expresiones lingüísticas, utilizadas como instrumentos en esa actividad (reglas semánticas, sintácticas, morfológicas y fonológicas).

La gramática funcional de Dik es «minimalista», ya que postula una sola representación sintáctica y semántica; es decir, en el caso de la representación sintáctica, ésta corresponde a la forma evidente; no 
hay representaciones abstractas como las hay en los niveles subyacentes de government and binding (Chomsky, 1981). Entre los niveles de representación de la GF hay un mapeo directo.

Es también una teoría ascendente en donde el punto de partida lo constituyen los elementos léxicos de una lengua (predicados y términos), los cuales se van expandiendo por medio de diferentes elementos hasta conformar una estructura totalmente especificada.

El componente gramatical consta de tres subcomponentes fundamentales: el fondo, la estructura de la cláusula y el componente expresivo. Veamos brevemente en qué consiste cada uno de ellos.

\section{Fondo}

El fondo es un componente donde se reúnen los lexemas de una lengua. En él se encuentra el lexicón que contiene los predicados y términos básicos de una lengua y las reglas léxicas, a través de las cuales se forman predicados y términos derivados.

Los predicados y términos básicos son asimilados por el individuo en el aprendizaje de su lengua materna. Los predicados y términos derivados son creados por el hablante con base en las reglas morfológicas de su lengua.

Los términos son expresiones con potencial referencial, es decir, expresiones que pueden usarse para referirse a entidades en algún mundo; los predicados designan propiedades de tales entidades o relaciones entre ellas. Predicados y términos son el punto de partida para el siguiente componente de la gramática: la estructura de la cláusula.

\section{Estructura de la cláusula}

La teoría de Dik (1989 y 1997) concibe la cláusula como una entidad compuesta por una estructura de capas. Cada una de las capas está motivada semánticamente. Esta perspectiva supone la adopción de una estructura estratal de al menos tres niveles de la cláusula: predicación, proposición y cláusula. A su vez, la predicación puede subdividirse en nuclear, central y extendida. Cada estructura se inserta en un estadio superior, formando así niveles de mayor complejidad.
A continuación describimos en qué consiste cada nivel.

Un predicado, junto con los elementos obligatorios exigidos por él (argumentos) y con los elementos opcionales (satélites), forma una predicación. Cuando se insertan expresiones referenciales (términos) en las posiciones argumentales se produce una predicación nuclear. El contenido de una predicación nuclear deriva, entonces, del significado del predicado y del significado o significados de los argumentos que a él pertenecen y que desempeñan una función semántica determinada con respecto a ese predicado. Por ejemplo, el predicado dar exige tres constituyentes obligatorios pero puede aparecer con otros dos constituyentes opcionales, en la oración: Santiago el fin de semana le dio a su padre las herramientas para que arreglara el carro, donde el fin de semana y para que arreglara el carro son satélites o constituyentes opcionales.

La predicación nuclear define las relaciones semánticas fundamentales existentes en cualquier expresión lingüística, mediante las cuales se describe un estado de cosas (EdC); para el caso de dar, quien da (agente), lo que da (meta) y a quien lo da (beneficiario). Si a esta representación se le añaden especificaciones como el aspecto y el modo, se obtiene una predicación central. Dichas especificaciones se realizan mediante la adición de operadores $(\pi)$ y satélites $(\sigma)$, cuya función es aportar diferentes modificaciones en cada uno de los estadios, contribuyendo de modo expansivo a la configuración de una cláusula. Tanto los operadores como los satélites son elementos opcionales, es decir, no requeridos por el predicado. La diferencia entre ellos es que los primeros son de carácter gramatical y los segundos de naturaleza léxica (García, 2003, p. 87), por ejemplo las marcas de aspecto (perfectivo/imperfectivo) frente a un constituyente como el fin de semana.

$\mathrm{Si}$ a la predicación central se le adicionan especificaciones temporales, espaciales y cognitivas de carácter externo al estado de cosas (por ejemplo, indicaciones de lugar, tiempo, cuantificación, modalidad) y funciones sintácticas, se obtiene una predicación extendida. En la oración Juan ama a María, al adicionar un satélite de modo como muchísimo, 
nos encontramos con una predicación extendida. La predicación extendida designa un estado de cosas, que puede ser ubicado en el espacio y en el tiempo. A partir de ella se puede construir una proposición, que designa un hecho posible. Los hechos posibles pueden ser evaluados en función de su veracidad o falsedad, pueden ser creídos, negados, recordados, etc. pero, al contrario que los EdC, no pueden ser localizados en el espacio o en el tiempo (Hengeveld, 1989, p. 132).

La asignación de funciones pragmáticas da inicio al nivel interpersonal y al siguiente nivel, el de la cláusula que designa un acto de habla. El acto de habla puede ser evaluado en función de su pertinencia en el proceso de interacción verbal, ya que especifica las intenciones comunicativas del hablante.

Cada estrato tiene un tipo de entidad, un orden de aparición y una representación por medio de una variable, tal como se muestra en el siguiente esquema²:

\begin{tabular}{|l|l|l|l|}
\hline Unidad Estructural & Tipo de entidad & Orden & Variable \\
\hline Predicado & $\begin{array}{l}\text { Propiedad/ } \\
\text { relación }\end{array}$ & 0 & F \\
\hline Término & Entidad & 1 & X \\
\hline Predicación & Estado de cosas & 2 & E \\
\hline Proposición & Hecho posible & 3 & X \\
\hline Cláusula & Acto de habla & 4 & E \\
\hline
\end{tabular}

Como hemos visto, el elemento central de la oración es el predicado, ya que desde su léxico determina qué tipo de elementos deben asociarse con él y qué características tienen estos elementos. Los predicados no se almacenan en el componente léxico de la lengua de manera aislada, sino asociados a las estructuras sintácticas de complementación a que dan lugar. Estas estructuras adquieren la forma de los llamados marcos predicativos.

Un marco predicativo consta de un predicado y un conjunto de argumentos. El número de argumentos está determinado por la valencia cuantitativa del predicado y el tipo de argumento por la valencia

2 Este esquema ha sido adaptado de García (2003, p. 86). cualitativa (qué función semántica cumplen dichos argumentos). De otra parte, el marco predicativo proporciona la siguiente información sobre un predicado: su forma léxica (por ejemplo si se trata de un verbo, si aparece en infinitivo, conjugado, en gerundio, etc.), la categoría sintáctica a la que pertenece, el número de argumentos que requiere, las restricciones léxicas de selección que establece el predicado sobre sus argumentos, las funciones semánticas que realizan los argumentos.

Veamos, por ejemplo, el marco predicativo del verbo amar:

Amar v [/+humano/]agente $[/+$ animado/ $]$ paciente

Así pues, el marco predicativo recoge las propiedades combinatorias y el significado de cada una de las piezas léxicas que constituyen el léxico y definen las propiedades semánticas fundamentales de la expresión lingüística en la que figuran, mediante las cuales se describe un estado de cosas (EdC).

Los estados de cosas pueden dividirse en cuatro tipos ${ }^{3}$, según dos parámetros diferenciadores: dinamismo y control. Un estado de cosas ( EdC) es dinámico cuando de un modo u otro implica una transición de una situación S1 a otra S2. Ejemplos: Juan abrió la puerta/el árbol cayó. En contraposición, un EdC es no dinámico cuando no implica ningún cambio, es decir, las entidades implicadas son las mismas en todos los puntos de la expresión de tiempo o durante la vigencia del EdC. Ejemplos: la mesa es roja/Juan permaneció en el hotel.

Un EdC tiene el rasgo de control cuando una de las entidades implicadas es controlador, esto es, tiene la capacidad de determinar si ese EdC sucederá o no. Por ejemplo, en la expresión «Juan abrió la puerta» se evidencia el rasgo de control, pues «Juan» puede abrir o no la puerta. En cambio, en «la mesa es roja», «la mesa» no tiene esa posibilidad y por lo tanto la expresión señala un EdC carente de control. De acuerdo con estos parámetros, tenemos 4 tipos básicos de EdC:

3 La tipología que a continuación se presenta ha sido retomada literalmente de Dik (1997). Los ejemplos también son suyos. Algunos lingüistas han cuestionado esta clasificación. 
- Estado: situación no controlada. Estado de cosas no dinámico. Juan es gordo.

- Proceso: estado de cosas dinámico y no controlado. Juan se cayó.

- Posición: estado de cosas dinámico, situación controlada. Juan permanecía en su silla.

- Acción: estado de cosas dinámico y controlado. Juan corría por el parque.

La tipología de los EdC provee las bases para la distinción entre argumentos y satélites, y sirve para determinar las funciones nucleares semánticas. Así mismo ayuda a definir el tipo de satélite que puede ir con un predicado, como lo veremos más adelante.

\section{Componente expresivo}

Este componente determina las reglas de expresión que dan paso a las expresiones lingüísticas. Estas reglas hacen referencia a fenómenos como la forma en que están realizados los términos (ya sea mediante marcas de casos, preposiciones, adposiciones, etc.), la forma en que se realiza el predicado mismo (específicamente, diferencias de voz en el verbo, elementos auxiliares, concordancia), el orden de los constituyentes y la prosodia (asignación de acento y entonación). Por ejemplo, en este componente la gramática funcional se ocupa de explicar las variaciones en el orden de una oración como Juan come pescado/pescado come Juan/come Juan pescado y de los fenómenos fonéticos y pragmáticos asociados a dicha variación.

Hemos descrito en términos generales el modelo que propone Dik para el componente gramatical de la lengua; a continuación presentamos algunos principios metodológicos.

\section{Principios metodológicos de la gramática funcional}

A continuación se indican los procedimientos que se emplean en la gramática funcional para determinar las funciones semánticas, los rasgos del predicado, el tipo de predicado y su valencia y el marco o marcos predicativos de un predicado.

\section{Procedimientos para determinar las funciones semánticas}

Para determinar las funciones semánticas se utilizan dos tipos de criterios. Criterios que la propia lengua emplea para marcar las funciones, esto es, los morfemas específicos, tales como sufijos, casos, preposiciones y conjunciones, y los rasgos léxicos de los términos (+humano, -animado, etc.). El análisis se hace con base en el contenido léxico y las marcas formales de los propios elementos. Sin embargo, como no siempre hay una relación unívoca entre una marca y una función, pues por un lado una misma función puede ser desempeñada por elementos muy diversos (por ejemplo, en latín el modo puede marcarse por medio de adverbios, a través del ablativo o con pro + ablativo) y a su vez una marca cumplir diversas funciones (tal como pro + ablativo, que puede indicar beneficiario, modo, dirección, ubicación, etc.), es necesario utilizar criterios indirectos como los que se exponen a continuación.

1. Fenómenos sintácticos que dependen del valor funcional de los términos que componen las predicaciones. Estos fenómenos son:

1.1 La interrogación parcial ${ }^{4}$ : una interrogación introducida por medio de un pronombre, adjetivo o adverbio interrogativo constituye un criterio de caracterización funcional, en la medida en que

una pregunta parcial se formula sobre un constituyente de la oración del que se conoce la función [...] pero del que se desconoce el contenido léxico.

En consecuencia, la respuesta a este tipo de preguntas ha de estar constituida, necesariamente, por un término que aporte la información semántica por la que se interroga, pero que desempeña la función que tal elemento tiene asignada en la predicación básica que constituye la pregunta (Villa, 1989, p. 295).

En la pregunta ¿quién rompió el vidrio?, se indaga por el agente de la acción. El constituyente respuesta tendrá, por tanto, la función de agente.

4 Sobre la validez de estos criterios, cf. Pinkster (1983), Villa (1987). 
1.2 La coordinación: según Dik (1997) y Pinkster (1983), en una predicación sólo pueden aparecer coordinados dos o más elementos que desempeñan la misma función y se encuentran en el mismo nivel sintáctico. En consecuencia, la coordinación implica que los dos términos afectados por ella son funcionalmente equivalentes.

La negación de la coordinación, esto es la yuxtaposición, constituye otro procedimiento de caracterización funcional: dos términos que aparecen yuxtapuestos han de expresar funciones distintas, puesto que una misma función no puede ser expresada dos veces en una misma predicación.

Estos criterios surgen al constatarse que cada función aparece sólo una vez en un mismo nivel de jerarquía, lo que implica que si una función está desempeñada por más de un elemento, este debe aparecer coordinado o en aposición. La presencia de elementos yuxtapuestos es un indicador de funciones distintas o de funciones en un nivel distinto de jerarquía. Este principio sirve para demostrar la identidad o no identidad funcional de un elemento. Por ejemplo, si se quiere adicionar otro elemento a la oración «Juan come manzanas» se empleará la coordinación (Juan come manzanas y peras/Juan y Pedro comen manzanas) y de esta manera se estará indicando que comparten la misma casilla argumental. En una oración como «Juan come manzanas todos los días en el colegio», la yuxtaposición del sintagma «todos los días» $\mathrm{y}$ «en el colegio», indica que tienen funciones distintas: frecuencia vs. ubicación.

Es necesario tener en cuenta que, en ocasiones, en una oración pueden aparecer términos aparentemente yuxtapuestos como en el caso de (18):

(18) Juan come manzanas, peras, mandarinas, zapotes y peras.

En (18) aparecen yuxtapuestos los sintagmas manzanas, peras, mandarinas, zapotes, lo que podría llevarnos a afirmar que tienen distinta función; sin embargo cuando los elementos son formalmente similares, ha de entenderse que acá hay una omisión (por razones estilísticas) del marcador de la coordinación.
1.3 La posibilidad de conmutación: este criterio sirve para demostrar que una misma función semántica o sintáctica puede ser expresada por marcas distintas, si la conmutación no altera la estructura sintáctica o el contenido semántico del enunciado.

De otra parte, como se ha señalado en Ochoa (2009), las funciones de los argumentos están determinadas por los rasgos léxicos del predicado; por ejemplo, que el segundo argumento de un verbo como «salir» desempeñe la función semántica de origen no depende más que de las características específicas de ese verbo. No obstante, en ocasiones, la función semántica de un argumento puede estar condicionada no sólo por el predicado, sino también por las características léxicas y funcionales de otros argumentos (Villa, 2003, p. 27). En general, es posible afirmar que en un predicado bivalente la función de un argumento puede ser distinta según el rasgo léxico del primer argumento /+/-animado/. Esto es lo que ocurre, por ejemplo, con el verbo «comer» si el primer argumento es /+humano/, la función del primer argumento será la de agente y la del segundo argumento será de meta, mientras que si el primer argumento es /-animado/, la función del primer argumento será de fuerza y la del segundo de meta en oraciones como Juan come pescado todos los días/ la sal se está comiendo la pared.

Algo similar, aunque en distinto grado, ocurre con los satélites: algunos están determinados por el predicado y otros por el conjunto del EdC. De acuerdo con Pinkster (1995, p. 37) los satélites de modo, instrumento y grado dependen sólo del predicado; en cambio satélites de lugar, tiempo, causa y circunstancia se relacionan con todo el EdC.

Es importante tener en cuenta que ciertos satélites son, a menudo, por lo que respecta a su aparición, sensibles a las propiedades de control y dinamismo (Dik, 1997, p. 227); por ejemplo, los satélites de compañía son compatibles con posiciones, acciones y procesos pero no con estados (Juan fue a la fiesta con María ${ }^{*}$ Juan es con María). De igual manera la presencia de satélites con las funciones semánticas de instrumento, beneficiario, fin y, en parte, modo están limitadas a las predicaciones nucleares con- 
troladas y/o dinámicas (lo mató con un revolver/* se sonrojó con un revolver). Las predicaciones nucleares no momentáneas pueden ser expandidas con satélites que denotan duración, mientras que con predicaciones nucleares télicas esto no siempre es posible (Dionisio fue tirano durante treinta y ocho años/*terminó el trabajo durante tres meses). Las predicaciones nucleares dinámicas, por otra parte, pueden ampliarse por medio de satélites que indican el tiempo consumido en producir el cambio («en cuánto tiempo»), por ejemplo: él llegó a la ciudad en poco tiempo.

Finalmente, para la asignación de funciones semánticas y para la descripción de los rasgos léxicos de los argumentos se tiene en cuenta el concepto de prototipo ${ }^{5}$. Como se sabe, las funciones semánticas y las caracterizaciones léxicas no son cerradas ni son discretas (Villa, 2003, p. 42). De esta forma, la asignación de un rasgo implica que el término en cuestión funciona como el más representativo o prototípico.

De acuerdo con el concepto de prototipo, las categorías de análisis se ven como un continuum en el que se perciben distintos grados: en un extremo se encuentra el elemento o ejemplo más representativo o prototipo y en el otro el menos representativo; en el medio hay distintas zonas de transición.

\section{Tipo de constituyente y valencia}

Una de las primeras decisiones que hay que tomar cuando se hace el análisis de un predicado es la de analizar si los constituyentes que aparecen con él son argumentos o satélites. Por ejemplo, en la oración en esto no te perdono, el constituyente en esto parece oscilar entre un argumento-meta o un satélite de referencia. La cuestión es, en gran medida, convencional, pues, como defiende Villa (2003, p. 26), entre ellos no hay una diferencia cualitativa, sino gradual. En cualquier caso, para determinar el carácter de un constituyente, los datos estadísticos son fundamentales: así si un predicado suele aparecer típicamente con ciertos constituyentes, es esperable que funcionen como argumentos; en cambio constituyentes

5 Sobre este concepto, véase a Lakoff (1987) y Langacker (1991a y b). que aparecen esporádicamente es más posible que funcionen como satélites.

El número de argumentos que puede tener un predicado resulta también problemático. Algunos predicados presentan datos diversos en este punto, no sólo cuando de forma clara proyectan estructuras diferentes, sino en otros en los que esporádicamente un verbo, por ejemplo, intransitivo (cantar) explicita un complemento interno (una canción). Al contrario, a veces elementos obligatorios son muy frecuentemente omitidos por razones contextuales, es decir, porque la información ya se conoce o se ha presentado previamente. Por ejemplo es posible que alguien diga Juan llega hoy sin necesidad de explicitar el punto de origen ni en el punto de llegada.

También un argumento puede omitirse si el predicado se emplea en forma absoluta. Una estructura absoluta es una estructura donde el complemento no aparece y no es recuperable de ninguna manera, pues el verbo está empleado en sentido genérico. En una frase como no es conveniente comer tanto no es necesario especificar el segundo argumento, pues se refiere a comer todo o mejor, comer cualquier cosa.

Para determinar la valencia cuantitativa de un predicado no parece disparatado acudir al criterio de la frecuencia: en un corpus de datos amplio y representativo, parece que el número de constituyentes que más frecuentemente se documentan con un predicado permitirá establecer su valencia. Por ejemplo el verbo amar en español aparece prototípicamente con dos constituyentes obligatorios.

De otra parte, la conceptualización del predicado puede dar indicios acerca del número de constituyentes obligatorios de dicho predicado. Por ejemplo el verbo perdonar conceptualmente presupone al menos dos entidades: quien perdona y a quien se perdona; por lo tanto, no es infundado pensar que tenga dos argumentos. Esto no significa que no pueda tener una proyección triple.

\section{Procedimientos para determinar los rasgos de un predicado}

De acuerdo con Dik, un predicado tiene control si es susceptible de designar mandatos o ruegos y promesas (corre, por favor, llega temprano, asistiré a 
la reunión). Todo predicado susceptible de aparecer en forma imperativa, en un acto de habla promisivo o dependiente de un ruego o solicitud generalmente designa estados de cosas controlados pues suponen que la entidad a la que se le ordena o ruega algo o quien hace una promesa tiene control sobre esa determinada situación.

En relación con el rasgo de dinamismo es preciso señalar que no hay criterios claros que permitan caracterizar un predicado en esta dirección.

\section{Procedimientos para determinar el número de marcos predicativos de un predicado}

Para poder determinar el marco -o los marcospredicativo de un verbo, se tiene en cuenta, inicialmente, la tipología de estado de cosas descrito por Dik, con base en los criterios de dinamicidad $\mathrm{y}$ control, esto es acciones, procesos, posiciones y estados; posteriormente, las características de los argumentos y de los términos que ocupan dichas posiciones argumentales.

Por otra parte, de acuerdo con Villa (2003), para determinar en qué condiciones un predicado tiene más de un marco predicativo ha de tenerse en cuenta que:

a. La variación en la valencia cuantitativa por sí sola no da lugar a varios marcos predicativos. Piénsese por ejemplo en el verbo perdonar en la oración en esto no te perdono frente $a$ te perdono. Sólo si la variación en la valencia va asociada a cambios en el significado del predicado, que no pueden reducirse a una regla más amplia, tendremos que reconocer varios marcos predicativos.

b. La diferencia de valencia cualitativa sólo da lugar a diferentes marcos predicativos, si no puede reducirse a un solo esquema.

c. Las diferencias en las restricciones léxicas sólo dan lugar a diferentes marcos predicativos si hay diferencia, además, en el significado del verbo o en la valencia cualitativa. En el caso que habíamos citado anteriormente entre Juan come pescado/la sal se está comiendo la pared, el verbo en la segunda oración cambia de significado y se acerca al del verbo corroer.
Entonces, para que un verbo tenga más de un marco predicativo es necesario que reúna algunas de las siguientes condiciones:

- Que describa cada uno un estado de cosas distinto.

- Que tenga el predicado distintos rasgos semánticos.

- Que sea diferente el léxico de los argumentos.

- Que presente más de una estructura.

Si el predicado presenta más de un marco predicativo, es necesario analizar si hay alguna razón de orden diacrónico, diafásico, dialectal, etc., que explique la coexistencia de estos marcos.

Para terminar, es importante señalar que a pesar de los procedimientos metodológicos anteriores, al momento de analizar un corpus concreto pueden presentarse dudas, pues, algunos de los criterios no son aplicables o son útiles para un tipo de elemento y no para otro. Por ejemplo, para identificar la función semántica no siempre aparecen los constituyentes en estructuras de coordinación. Ocurre también que los datos estadísticos no permiten en ocasiones discernir, por ejemplo, entre un argumento y un satélite. Es preciso en estos casos recurrir al criterio del investigador y al de los hablantes.

\section{La gramática del rol y la referencia}

En forma casi paralela al trabajo de Dik y al de sus seguidores, en Norteamérica se ha desarrollado el modelo conocido como gramática del rol y la referencia. En este modelo se pone un gran peso en las funciones semánticas y en el carácter referencial de las oraciones, de ahí el nombre del modelo (del papel y la referencia), sin desconocer el papel esencial de la pragmática y la sintaxis.

Este modelo teórico conocido como funcionalismo norteamericano ha sido desarrollado principalmente por Van Valin, Folley y Van Valin, y Van Valin y La Polla. Estos autores ponen un gran peso en las funciones semánticas y en el carácter referencial de las oraciones, de ahí el nombre del modelo (del papel y la referencia).

$\mathrm{Al}$ igual que en el modelo de Dik, destacan la interrelación de los distintos niveles de la lengua, 
reconocen la determinación de factores cognitivos, semánticos y pragmáticos sobre la sintaxis, establecen una distinción entre predicado y argumento y entre argumento y no argumento. Hay también un trabajo importante a nivel de la descomposición lexical. A diferencia de Dik, sólo reconocen a nivel semántico dos macrofunciones o macropapeles: el de actor y el de experimentador; estos papeles pueden representarse en la estructura superficial de diversos modos, por ejemplo, el actor puede materializarse como agente e incluso como instrumento (Butler et al, 1999).

Los macroroles constituyen, entonces, una gama de relaciones temáticas graduadas a partir del principio de prototipicidad: de la más prototípica y cercana al macro rol (agente: +agentivo, +prominente, +animado) a la menos prototípica (paciente: +delimitado, +focalizado, +afectado).

Foley y Van Valin parten de una concepción intraclausal de la gramática, donde

distinguen dos niveles de representación: una estructura semántica que consiste en el predicado verbal con sus argumentos, y una estructura morfosintáctica que es el resultado de la evolución diacrónica pero, al mismo tiempo, está determinada por factores de tipo semántico, pragmático y sociolingüístico" (Butler et al, 1999:25)

La cláusula está representada por tres capas: el núcleo (verbo), el centro (núcleo + argumentos) y

\section{Bibliografía}

Butler, Ch., Mairal, R., Martín, J., Arista y Ruiz, F. (1999). Nuevas perspectivas en gramática funcional. Barcelona: Ariel lingüística.

Chomsky, N. (1981). Lectures on goverment and biding. Foris: Dordrecht.

Dik, S. C. (1978). Functional Grammar. Amsterdam: North- Holland.

(1989). The theory of functional grammar. Dordrecht: foris.

(1997). The theory of functional grammar. part 1. The structure of the clause. Berlin: Mouton de Gruyter. la periferia (incluye a los no argumentos, adjuntos). Los elementos modificadores son por un lado los adjuntos y por otro los operadores que conllevan información gramatical del tipo espacio, tiempo, fuerza ilocucionaria, negación, modalidad.

En este modelo se hace una diferenciación entre valencia sintáctica (valencia cuantitativa: numero de argumentos regidos) y valencia semántica: (valencia cualitativa: funciones semánticas). Estos dos tipos de valencia no siempre coinciden pues un verbo como golpear en la voz activa tiene dos argumentos sintácticos y dos semánticos, pero en la estructura pasiva sólo tiene un argumento sintáctico y dos funciones semánticas. Esta distinción es importante para explicar algunos fenómenos lingüísticos como la transitividad:

En la GRR la transitividad no está caracterizada en términos del número de argumentos sintácticos sino que está definida en términos del número de macro roles que un verbo toma. Las tres posibilidades de transitividad de macro roles (M-transitividad) son: transitiva (2 macro roles), intransitiva ( 1 macro roles) y atransitiva (0 macro roles) (Mora, 2005: 55).

El desarrollo del modelo de Dik y los trabajos de la gramática del rol y la referencia son, sin duda, los modelos lingüísticos más vigentes en la actualidad pues han hecho grandes aportaciones a la lingüística teórica, descriptiva y tipológica.

Dik. (s.f.). Oviedo: Universidad de Oviedo.

García, D. (2003). Funcionalismo y lingüística: la gramática funcional de S. C.

González, C. (2006). La gramática del papel y la referencia: una aproximación al modelo. Onomázein 14/2, p.p. 101-140.

Halliday, M. A. K. (1982). El lenguaje como semiótica social. México: Fondo de Cultura Económica (Trabajo original publicado en1967).

Hengeveld, K. (1989). Layers and operators. Journal of Linguistics, 25. Cambidge: Cambridge, University Press. 
Langacker, R. (1991a). Foundations of cognitive grammar II: descriptive application. Stanford: Stanford University Press.

Lakoff, G. (1987). Women, fire, and dangerous things. Chicago: The University of Chicago Press.

Langacker, R. (1991b). Cognitive Grammar. En: F. Droste and J. Joseph (Eds.). Linguistic theory and grammatical description nine current approaches (p.p. 275-306). Amsterdam: John Benjamins Publishing Company.

Martinet, A. (1978). Estudios de sintaxis funcional. Madrid: Gredos (Trabajo original publicado en1962).

Mora, A. (2005). La transitividad en construcciones oracionales constituidas por un verbo matriz y un objeto oracional subordinado introducido por «que». México: El Colegio de México (Tesis doctoral).

Pinkster, H. (1989). Latin linguistics and linguistic theory. Amsterdam: John Benjamins Publishing Company.

(1995). Semántica y sintaxis del latín. Madrid: Ediciones clásicas.

Tesniere, L. (1959). Èlements de syntaxe structurale. París: Klincksieck.
Ochoa, L. (2005). Estructuras semántico-sintácticas de algunos verbos de régimen dativo en latín. Tesis doctoral inédita. Madrid: Universidad Autónoma de Madrid.

(2009). Verbos latinos «intransitivos» de régimen dativo. Bogotá: Universidad Nacional de Colombia, Centro Editorial, Facultad de Ciencias Humanas.

Van Valin, R. (1993). A synopsis of role and reference grammar. En: Robert D. Van Valin, Jr. (Eds.): $A d$ vances in role and reference grammar. Amsterdam/ Philadelphia: John Benjamins, p.p. 1-164.

Villa, J. de la (1987). Las funciones de los elementos nominales: criterios para su identificación y caracterización en griego y latín. Cuadernos de Filología Clásica, 22. Madrid: Universidad Complutense de Madrid.

(2003). Límites y alternancias en los marcos predicativos. En: J. M. Baños et al. Praedicativa: complementación en griego y latín (p.p. 19-49). Santiago de Compostela: Universidad Santiago de Compostela. 